\title{
Synthesis and Characterization of Dimaleimide Fluorogens Designed for Specific Labeling of Proteins
}

\author{
Stéphane Girouard ${ }^{\ddagger}$, Marie-Hélène Houle ${ }^{\ddagger}$, Alain Grandbois ${ }^{\ddagger}$, \\ Jeffrey W. Keillor ${ }^{\ddagger \dagger} *$ and Stephen W. Michnick ${ }^{\dagger}$ \\ ${ }^{\ddagger}$ Department of Chemistry and ${ }^{\dagger}$ Department of Biochemistry, \\ Université de Montréal, C.P. 6128, Succ. centre- ville, \\ Montréal, Québec H3C 3J7 Canada
}

\section{Supporting Information}




\section{Experimental Section}

$\underline{\text { Synthesis }}$

\section{Materials}

The following material were obtained from Aldrich and were used without further purification: 2-hydroxy-5-nitrobenzaldehyde, $N$-acetylglycine, maleic anhydride, $p$-nitrophenylacetic acid, Laspartic acid dimethyl ester hydrochloride, citraconic anhydride, dimethyl L-tartrate, dimethyl malonate. All reactions were carried out under an atmosphere of dry nitrogen employing conventional bench-top techniques except for reactions with thiols. ${ }^{13} \mathrm{C}-\mathrm{NMR}$ and ${ }^{1} \mathrm{H}-\mathrm{NMR}$ spectra were recorded on AMXR400 or AMX300 spectrometers and were referenced to the residual proton or ${ }^{13} \mathrm{C}$ signal of the solvent. Mass spectra were determined by $\mathrm{FAB}+$ ionization on an AutoSpecQ spectrometer at the Regional Mass Spectrometry Centre at the Université de Montréal. Infrared spectra were recorded on a Perkin-Elmer (FTIR) spectrometer. Melting points (uncorrected) were determined on a Unimelt Tomas-Hoover or on a Gallenkamp melting point apparatus.

\section{Methods}

\section{3-Acetamido-6-nitrochromen-2-one (1)}

A dry 100-mL round bottomed flask was charged with $7.11 \mathrm{~g}(42.5 \mathrm{mmol})$ of 2-hydroxy-5nitrobenzaldehyde. To this flask were then added $N$-acetylglycine (4.98 $\mathrm{g}, 42.5 \mathrm{mmol})$ and acetic anhydride $(40.1 \mathrm{~mL}, 0.43 \mathrm{~mol})$. In small aliquots, $1.71 \mathrm{~g}(42.5 \mathrm{mmol})$ of sodium hydride (60\% dispersion in mineral oil) was added to the flask. Reactants were observed to dissolve and after 2-5 minutes, precipitation occurred. The reaction mixture was stirred for $20 \mathrm{~h}$ and $7.11 \mathrm{~mL}$ of water were added. Following the addition of $43 \mathrm{~mL}$ acetic acid, the mixture was cooled to 
$4^{\circ} \mathrm{C}$ for $4 \mathrm{~h}$. The resulting precipitate was filtered and washed liberally with cold glacial acetic acid. The acetic acid was then removed as an azeotrope upon addition of $250 \mathrm{~mL}$ toluene and rotary evaporation to dryness, three times. The final residue was dried under vacuum to give product 1 as a beige powder $(6.40 \mathrm{~g}, 25.8 \mathrm{mmol})$ in a yield of $61 \%$. mp $277-279{ }^{\circ} \mathrm{C}\left(\mathrm{lit}^{1} 278^{\circ} \mathrm{C}\right)$ FTIR (KBr) $\left(\mathrm{cm}^{-1}\right)$ 3350, 3050, 1710, 1680, 1600, 1500, 1420, and 1335. ${ }^{1} \mathrm{H}-\mathrm{NMR}$ (DMSO-d $\mathrm{d}_{6}$ ) : $\delta(\mathrm{ppm}) 9.93(\mathrm{~s}, 1 \mathrm{H}), 8.76(\mathrm{~s}, 1 \mathrm{H}), 8.73(\mathrm{~d}, J=2.5 \mathrm{~Hz}, 1 \mathrm{H}), 8.28(\mathrm{dd}, J=2.8 \mathrm{~Hz}, J=9.1 \mathrm{~Hz}$, 1H), $7.60(\mathrm{~d}, J=9.1 \mathrm{~Hz}, 1 \mathrm{H}), 2.19$ (s, 3H). ${ }^{13} \mathrm{C}-\mathrm{NMR} \quad\left(\mathrm{DMSO}_{\mathrm{d}}\right.$ ): $\delta$ (ppm) 171.47, 157.69, 145.07, 127.11, 124.96, 124.52, 122.79, 121.52, 118.26, 25.00. HRMS expected: 249.0524; found: 249.0534. Elemental analysis for $\mathrm{C}_{11} \mathrm{H}_{8} \mathrm{~N}_{2} \mathrm{O}_{5}$, expected: $\mathrm{C}, 53.23 ; \mathrm{H}, 3.25 ; \mathrm{N}, 11.27$; found: C, 53.19; H, 3.34; N, 11.06 .

\section{3-Acetamido-6-aminochromen-2-one (2)}

Palladium $10 \mathrm{wt} \%$ on activated carbon $\left(0.322 \mathrm{~g}, 1 / 10^{\text {th }}\right.$ the mass of $\left.\mathbf{1}\right)$ was placed in a $1-\mathrm{L}$ roundbottom flask and the flask was purged with nitrogen. A solution of sodium borohydride (1.21g, $32.9 \mathrm{mmol})$ in $22.6 \mathrm{~mL}$ water was added dropwise. A suspension of compound 1 ( $3.20 \mathrm{~g}, 12.9$ $\mathrm{mmol})$ in methanol $(775 \mathrm{~mL})$ was then added over a two hour period and allowed to stir at room temperature an additional $30 \mathrm{~min}$, when the reaction mixture was filtered through Celite and the solvent was removed by rotary evaporation. Water $(750 \mathrm{~mL})$ was added to the residue and the solution was cooled to $4^{\circ} \mathrm{C}$ for $20 \mathrm{~h}$. The resulting precipitate was filtered and rinsed with cold distilled water. The solid was recrystallized from ethanol to give 2 as a yellow solid (1.59 g, 7.3 mmol) in $57 \%$ yield. $\mathrm{mp} 250-252{ }^{\circ} \mathrm{C}\left(\operatorname{lit}^{2} 252-253{ }^{\circ} \mathrm{C}\right)$. FTIR $(\mathrm{KBr})\left(\mathrm{cm}^{-1}\right): 3410,3310$, 1700, 1650, 1630. ${ }^{1} \mathrm{H}-\mathrm{NMR}\left(\right.$ DMSO-d $\left._{6}\right): \delta(\mathrm{ppm}) 9.65(\mathrm{~s}, 1 \mathrm{H}), 8.40(\mathrm{~s}, 1 \mathrm{H}), 7.10(\mathrm{dd}, J=9.1$ $\mathrm{Hz}, J=2.7 \mathrm{~Hz}, 1 \mathrm{H}), 6.73(\mathrm{~d}, J=9.1 \mathrm{~Hz}, 1 \mathrm{H}), 6.68(\mathrm{~d}, J=2.7 \mathrm{~Hz}, 1 \mathrm{H}), 5.19(\mathrm{bs}, 2 \mathrm{H}), 2.14(\mathrm{~s}$, 
3H). ${ }^{13} \mathrm{C}-\mathrm{NMR}\left(\right.$ DMSO-d $\left._{6}\right): \delta(\mathrm{ppm}) 171.12,158.78,146.92,142.52,125.26,124.98,120.82$, 117.54, 117.15, 110.66, 24.93. HRMS expected, 219.0777; found, 219.0770. Elemental analysis for $\mathrm{C}_{11} \mathrm{H}_{10} \mathrm{~N}_{2} \mathrm{O}_{3}$ : expected : $\mathrm{C}, 60.55 ; \mathrm{H}, 4.62 ; \mathrm{N}, 12.84$; found : $\mathrm{C}, 60.15 ; \mathrm{H}, 4.50 ; \mathrm{N}$, 12.62 .

\section{3,6-Diaminochromen-2-one (3)}

To a solution composed of $12.63 \mathrm{~mL}$ of $12 \mathrm{M}$ hydrochloric acid in $6.32 \mathrm{~mL}$ ethanol was added $0.919 \mathrm{~g}(4.2 \mathrm{mmol})$ of compound 2. The solution was heated to reflux for $30 \mathrm{~min}$ and then cooled to $4{ }^{\circ} \mathrm{C}$ overnight. The resulting precipitate was removed by filtration and dissolved in a minimal volume of distilled water. The solution was neutralized by addition of $25 \%$ ammonium hydroxide and cooled at $4{ }^{\circ} \mathrm{C}$ for three hours. The resulting precipitate was removed by filtration and recrystallized from water to give a yellow compound $(3)(0.484 \mathrm{~g}, 2.7 \mathrm{mmol})$ in $65 \%$ yield. mp $183-186{ }^{\circ} \mathrm{C}\left(\mathrm{lit}^{1} 183-184^{\circ} \mathrm{C}\right)$. FTIR $(\mathrm{KBr})\left(\mathrm{cm}^{-1}\right): 3420,3340,1690,1635 .{ }^{1} \mathrm{H}-\mathrm{NMR}$ $\left(\right.$ DMSO-d $\left._{6}\right): \delta(\mathrm{ppm}) 6.95(\mathrm{~d}, J=8.5 \mathrm{~Hz}, 1 \mathrm{H}), 6.56(\mathrm{~s}, 1 \mathrm{H}), 6.49(\mathrm{~d}, J=2.4 \mathrm{~Hz}, 1 \mathrm{H}), 6.46(\mathrm{dd}, J$ $=10.0 \mathrm{~Hz}, J=2.6 \mathrm{~Hz}, 1 \mathrm{H}), 5.48(\mathrm{bs}, 2 \mathrm{H}), 5.00(\mathrm{bs}, 2 \mathrm{H}) .{ }^{13} \mathrm{C}-\mathrm{NMR}\left(\mathrm{DMSO}-\mathrm{d}_{6}\right): \delta(\mathrm{ppm})$ $159.95,146.50,141.19,134.13,123.04,116.75,113.70,109.30,108.42$. HRMS expected : 177.0592; found : 177.0598 .

\section{3,6-Dimaleimidylchromen-2-one (4)}

Diamine 3 (200 mg, $1.14 \mathrm{mmol}$ ) and maleic anhydride (335 mg, $3.42 \mathrm{mmol})$ were placed in a dry 50-mL round bottom flask. Chloroform $(11.5 \mathrm{~mL})$ was added and the solution was heated to reflux for $20 \mathrm{~h}$. The mixture was then filtered and the recovered solid was rinsed liberally with chloroform and then dried under vacuum. To this solid was added acetic anhydride $(9 \mathrm{~mL}, 96$ 
mmol) and sodium acetate $(35 \mathrm{mg}, 0.427 \mathrm{mmol}$ ) and the reaction was stirred vigorously for another $30 \mathrm{~min}$. The mixture was then cooled to $4{ }^{\circ} \mathrm{C}$ for $4 \mathrm{~h}$ and filtered. The beige solid thus obtained was dried over vacuum to give compound 4 in $20 \%$ yield (76 mg, $0.226 \mathrm{mmol}$ ). $\mathrm{mp}$ : $>296{ }^{\circ} \mathrm{C}(\mathrm{dec})$. FTIR $(\mathrm{KBr})\left(\mathrm{cm}^{-1}\right): 1700,1620 .{ }^{1} \mathrm{H}-\mathrm{NMR}\left(\mathrm{CDCl}_{3}\right): \delta(\mathrm{ppm}) 7.78(\mathrm{~s}, 1 \mathrm{H}), 7.66$ $(\mathrm{dd}, J=9.8 \mathrm{~Hz}, J=2.5 \mathrm{~Hz}, 1 \mathrm{H}), 7.63(\mathrm{~d}, J=2.5 \mathrm{~Hz}, 1 \mathrm{H}), 7.50(\mathrm{~d}, J=8.7 \mathrm{~Hz}, 1 \mathrm{H}), 6.93(\mathrm{~s}, 2 \mathrm{H})$, $6.91(\mathrm{~s}, 2 \mathrm{H}) .{ }^{13} \mathrm{C}-\mathrm{NMR}\left(\mathrm{DMSO}-\mathrm{d}_{6}\right): \delta(\mathrm{ppm}) 1699.99,169.06,156.80,151.50,143.62,135.82$ 134.89, 131.61, 128.63, 127.11, 118.60, 118.13, 117.58. HRMS : expected, 337.0463; found, 337.0461.

\section{3,6-Di-(3'-methoxycarbonylmaleimidyl)chromen-2-one (5)}

To a suspension of diamine $\mathbf{3}(268 \mathrm{mg}, 1.52 \mathrm{mmol})$ in dry chloroform $(15.4 \mathrm{~mL})$ was added á carbomethoxymaleic anhydride $(712 \mathrm{mg}, 4.56 \mathrm{mmol})$. The solution was heated to reflux for 20 $\mathrm{h}$, then cooled to room temperature and filtered. The solid thus obtained was rinsed liberally with chloroform and dried under reduced pressure. It was then added to $12 \mathrm{~mL}$ acetic anhydride and $47 \mathrm{mg}$ of sodium acetate $(0.56 \mathrm{mmol})$. The mixture was then heated to $100{ }^{\circ} \mathrm{C}$ for $90 \mathrm{~min}$ and then allowed to cool to room temperature for $24 \mathrm{~h}$. The resulting precipitate was recovered by filtration and rinsed with water, giving the desired product 5 in $22 \%$ yield $(151 \mathrm{mg}, 0.33$ mmol). $\mathrm{mp}>230{ }^{\circ} \mathrm{C}(\mathrm{dec})$. FTIR $(\mathrm{KBr})\left(\mathrm{cm}^{-1}\right): 1720,1670 .{ }^{1} \mathrm{H}-\mathrm{NMR}\left(\mathrm{DMSO}-\mathrm{d}_{6}\right): \delta(\mathrm{ppm})$ $8.24(\mathrm{~s}, 1 \mathrm{H}),, 8.04(\mathrm{~s}, 2 \mathrm{H}), 7.66(\mathrm{dd}, J=8.97 \mathrm{~Hz}, J=2.43 \mathrm{~Hz}, 1 \mathrm{H}), 7.42(\mathrm{~d}, J=8.96 \mathrm{~Hz}, 1 \mathrm{H})$ $7.40(\mathrm{~d}, J=2.42 \mathrm{~Hz}, 1 \mathrm{H}), 4.04(\mathrm{~s}, 3 \mathrm{H}), 4.00(\mathrm{~s}, 3 \mathrm{H}) .{ }^{13} \mathrm{C}-\mathrm{NMR}\left(\mathrm{DMSO}_{\mathrm{d}}\right): \delta(\mathrm{ppm}) 171.02$, $163.38,132.09,159.92,145.83,142.80,142.31,135.32,128.06,124.83,120.92,119.92,119.27$, 117.16, 48.83. HRMS expected for $\mathrm{C}_{21} \mathrm{H}_{12} \mathrm{~N}_{2} \mathrm{O}_{10}: 452.0492$; found: 452.0491 . 


\section{3,6-Di-(3' -methylmaleimidyl)chromen-2-one (6)}

To a suspension of diamine 3 (268 $\mathrm{mg}, 1.52 \mathrm{mmol}$ ) in $15.4 \mathrm{~mL}$ of dry chloroform was added citraconic anhydride $(410 \mu \mathrm{L}, 4.56 \mathrm{mmol})$. The mixture was heated to reflux for $20 \mathrm{~h}$. The mixture was cooled to room temperature and filtered, and the recovered solid was rinsed well with chloroform. After drying, it was added to a solution of sodium acetate (47 mg, $0.56 \mathrm{mmol}$ ) in acetic anhydride $(12 \mathrm{~mL})$. The mixture was heated to $100{ }^{\circ} \mathrm{C}$ for $90 \mathrm{~min}$ and then cooled to room temperature and allowed to stand for $24 \mathrm{~h}$. The resulting precipitate was then filtered and rinsed with water to give the desired product 6 in $20 \%$ yield $(147 \mathrm{mg}, 0.3 \mathrm{mmol}) . \mathrm{mp}>230{ }^{\circ} \mathrm{C}$ (dec). FTIR $(\mathrm{KBr})\left(\mathrm{cm}^{-1}\right): 1720,1654 .{ }^{1} \mathrm{H}-\mathrm{NMR}\left(\mathrm{CDCl}_{3}\right): \delta(\mathrm{ppm}) 8.52(\mathrm{~s}, 1 \mathrm{H}), 7.88(\mathrm{~d}, J=$ $2.29 \mathrm{~Hz}, 1 \mathrm{H}), 7.63(\mathrm{~s}, 1 \mathrm{H}), 7.52(\mathrm{dd}, J=9.26 \mathrm{~Hz}, J=2.29 \mathrm{~Hz}, 1 \mathrm{H}), 7.45(\mathrm{~s}, 1 \mathrm{H}), 7.34(\mathrm{~d}, J=$ $8.83 \mathrm{~Hz}, 1 \mathrm{H}), 2.07(\mathrm{~d}, J=6.92 \mathrm{~Hz}, 3 \mathrm{H}), 1.91(\mathrm{~d}, J=7.02 \mathrm{~Hz}, 3 \mathrm{H}) .{ }^{13} \mathrm{C}-\mathrm{NMR}\left(\mathrm{DMSO}-\mathrm{d}_{6}\right): \delta$ (ppm) 170.00, 169.02, 158.90, 146.22, 137.32, 126.04, 122.06, 120.43, 118.24, 117.16, 24.80. HRMS expected for $\mathrm{C}_{19} \mathrm{H}_{12} \mathrm{~N}_{2} \mathrm{O}_{6}: 364.0695$; found: 364.0693 .

\section{3,6-Di(3'-ethylthiosuccinimidyl)chromen-2-one (7)}

To a suspension of 4 ( $40 \mathrm{mg}, 0.12 \mathrm{mmol})$ in $3 \mathrm{~mL}$ dry chloroform was added $27 \mu \mathrm{L}(0.36 \mathrm{mmol})$ of ethanethiol. The solution was stirred at room temperature for $12 \mathrm{~h}$, then concentrated under reduced pressure and purified by flash chromatography (30:70 EtOAc:hexane). Compound 7 was thus obtained as a yellow solid in $85 \%$ yield ( $47 \mathrm{mg}, 0.10 \mathrm{mmol}$ ). $\mathrm{mp} 128-131{ }^{\circ} \mathrm{C}$. FTIR ( $\mathrm{KBr})\left(\mathrm{cm}^{-1}\right): 3000,1700,1620,600 .{ }^{1} \mathrm{H}-\mathrm{NMR}\left(\mathrm{CDCl}_{3}\right): \delta(\mathrm{ppm}) 7.46(\mathrm{~m}, 2 \mathrm{H}), 7.42(\mathrm{~d}, J=$ $11.0 \mathrm{~Hz}, 1 \mathrm{H}) 7.39(\mathrm{~s}, 1 \mathrm{H}), 3.90(\mathrm{~d}, J=5.6 \mathrm{~Hz}, 1 \mathrm{H}), 3.88(\mathrm{~d}, J=5.6 \mathrm{~Hz}, 1 \mathrm{H}), 3.35(\mathrm{q}, J=9.7 \mathrm{~Hz}$, 2H), $2.94(\mathrm{~m}, 2 \mathrm{H}), 2.80(\mathrm{~m}, 2 \mathrm{H}), 2.69(\mathrm{~d}, J=5.6 \mathrm{~Hz}, 1 \mathrm{H}), 2.64(\mathrm{~d}, J=5.6 \mathrm{~Hz}, 1 \mathrm{H}), 1.29(\mathrm{t}, J=$ $8.0 \mathrm{~Hz}, 6 \mathrm{H}) .{ }^{13} \mathrm{C}-\mathrm{NMR}\left(\mathrm{CDCl}_{3}\right): \delta(\mathrm{ppm}) 175.20,173.42,167.90,155.98,151.07,136.55$, 
$130.14,128.85,128.25,125.39,118.72,117.35,38.72,35.96,26.04,20.40,14.03$. HRMS calculated: 461.0851; found: 461.0849 . Elemental analysis for $\mathrm{C}_{21} \mathrm{H}_{20} \mathrm{~N}_{2} \mathrm{O}_{6} \mathrm{~S}_{2}$ expected: $\mathrm{C}$, 54.77; H, 4.38; N, 6.08; S, 13.92; found: C, 54.49; H, 4.35; N, 5.99; S, 13.50.

\section{3,6-Di-(3'-ethylthio-4'-methoxycarbonylsuccinimidyl)chromen-2-one (8)}

The reaction of $54 \mathrm{mg}(0.12 \mathrm{mmol})$ of compound 5 in $3 \mathrm{~mL}$ dry DMSO with $27 \mu \mathrm{L}$ ethanethiol (0.36 mmol) was carried out according to the same protocol used to obtain compound $\mathbf{7}$, giving compound 8 in $83 \%$ yield $(58 \mathrm{mg}, 0.1 \mathrm{mmol})$. FTIR $(\mathrm{KBr})\left(\mathrm{cm}^{-1}\right): 3000,1727,1622,600 .{ }^{1} \mathrm{H}-$ NMR (DMSO-d 6 ) : $\delta(\mathrm{ppm}) 8.24(\mathrm{~s}, 1 \mathrm{H}), 7.53(\mathrm{~d}, J=2.63,1 \mathrm{H}), 7.36(\mathrm{dd}, J=9.24 \mathrm{~Hz}, J=2.67$ $\mathrm{Hz}, 1 \mathrm{H}), 7.24(\mathrm{~d}, J=9.24 \mathrm{~Hz}, 1 \mathrm{H}), 4.38(\mathrm{~d}, 1 \mathrm{H}), 4.32(\mathrm{~s}, 1 \mathrm{H}), 4.00(\mathrm{~s}, 3 \mathrm{H}), 3.98(\mathrm{~d}, J=5.6 \mathrm{~Hz}$, $3 \mathrm{H}), 3.92(\mathrm{~d}, J=5.4 \mathrm{~Hz}, 1 \mathrm{H}), 3.87(\mathrm{~d}, J=5.6 \mathrm{~Hz}, 1 \mathrm{H}), 2.80(\mathrm{~m}, 4 \mathrm{H}), 1.30(\mathrm{t}, J=7.24 \mathrm{~Hz}, 6 \mathrm{H})$. ${ }^{13}$ C-NMR (DMSO-d 6 ) : $\delta(\mathrm{ppm}) 175.06,172.67,168.06,158.44,150.63,137.28,128.83,128.32$ 124.22, 119.69, 118.47, 117.21, 50.01, 46.23, 42.16, 39.12, 14.24, 12.02. HRMS expected for $\mathrm{C}_{25} \mathrm{H}_{24} \mathrm{~N}_{2} \mathrm{O}_{10} \mathrm{~S}_{2}: 576.0872$; found: 576.0874 .

\section{3,6-Di-(3'-ethylthio-4'-methylsuccinimidyl)chromen-2-one (9)}

The reaction of $44 \mathrm{mg}(0.12 \mathrm{mmol})$ of compound 6 in $3 \mathrm{~mL}$ dry DMSO with $27 \mu \mathrm{L}$ ethanethiol (0.36 mmol) was carried out according to the same protocol used to obtain compound $\mathbf{7}$, giving compound 9 in $84 \%$ yield $(49 \mathrm{mg}, 0.1 \mathrm{mmol})$. FTIR $(\mathrm{KBr})\left(\mathrm{cm}^{-1}\right):, 1720,1620,600 .{ }^{1} \mathrm{H}-\mathrm{NMR}$ $\left(\mathrm{CDCl}_{3}\right): \delta(\mathrm{ppm}) 8.21(\mathrm{~s}, 1 \mathrm{H}), 7.57(\mathrm{~d}, J=2.53 \mathrm{~Hz}, 1 \mathrm{H}), 7.39(\mathrm{dd}, J=8.47 \mathrm{~Hz}, J=2.67 \mathrm{~Hz}$, 1H), $7.26(\mathrm{~d}, J=8.35 \mathrm{~Hz}, 1 \mathrm{H}), 3.84(\mathrm{~s}, 1 \mathrm{H}), 3.12(\mathrm{~s}, 1 \mathrm{H}), 3.07(\mathrm{~s}, 1 \mathrm{H}, 2.83(\mathrm{~m}, 4 \mathrm{H}), 1.93(\mathrm{~d}, J=$ $7.08 \mathrm{~Hz}, 3 \mathrm{H}), 1.90(\mathrm{~d}, J=7.25 \mathrm{~Hz}, 3 \mathrm{H}), 1.30(\mathrm{t}, J=7.28 \mathrm{~Hz}, 6 \mathrm{H}) \cdot{ }^{13} \mathrm{C}-\mathrm{NMR}\left(\right.$ DMSO-d $\left.\mathrm{d}_{6}\right): \delta$ (ppm) 175.04, 172.32, 167.92, 156.34, 150.73, 137.21, 129.87, 128.65, 124.34, 118.03, 117.04, 
38.53, 34.29, 26.02, 20.68, 14.27, 11.67. HRMS expected for $\mathrm{C}_{23} \mathrm{H}_{24} \mathrm{~N}_{2} \mathrm{O}_{6} \mathrm{~S}_{2}$ : 488.1076; found: 488.1072.

\section{3,6-(4,4'-bis(methylmercapto-3-succinimidyl)benzophenone)chromen-2-one (10)}

Compound $4(42 \mathrm{mg}, 0.13 \mathrm{mmol})$ and BMMB $(34 \mathrm{mg}, 0.13 \mathrm{mmol})$ were placed in a $10-\mathrm{mL}$ round bottom flask. Chloroform $(2.5 \mathrm{~mL})$ was then added with stirring. To this suspension triethylamine ( $52 \mu \mathrm{L}, 0.39 \mathrm{mmol}$ ) was then added and the reaction mixture was heated to reflux for $12 \mathrm{~h}$. The mixture was cooled to room temperature. A $50-\mathrm{mL}$ aliquot of water was then added and the solution was extracted 4 times with $20 \mathrm{~mL}$ dichloromethane. The organic layers were combined, dried over $\mathrm{MgSO}_{4}$ and removed under reduced pressure. The resulting product was then purified by flash chromatography (20:80 EtOAc:hexane). Compound $\mathbf{1 0}$ was thus obtained as a beige solid in $78 \%$ yield $(61 \mathrm{mg}, 0.10 \mathrm{mmol}) . \mathrm{mp}:>220{ }^{\circ} \mathrm{C}(\mathrm{dec})$. FTIR $(\mathrm{KBr})$ $\left(\mathrm{cm}^{-1}\right): 1720,1654,1605,602 .{ }^{1} \mathrm{H}-\mathrm{NMR}\left(\mathrm{CDCl}_{3}\right): \delta(\mathrm{ppm}) 7.81(\mathrm{~d}, J=8.0 \mathrm{~Hz}, 4 \mathrm{H}), 7.73(\mathrm{~d}, J$ $=7.9 \mathrm{~Hz}, 4 \mathrm{H}), 7.60(\mathrm{~d}, J=9.4 \mathrm{~Hz}, 1 \mathrm{H}), 7.52(\mathrm{~s}, 1 \mathrm{H}), 7.45(\mathrm{~d}, J=9.6 \mathrm{~Hz}, 1 \mathrm{H}), 7.39(\mathrm{~s}, 1 \mathrm{H}), 4.40$ $(\mathrm{d}, J=5.6 \mathrm{~Hz}, 1 \mathrm{H}), 3.96(\mathrm{~d}, J=5.5 \mathrm{~Hz}, 1 \mathrm{H}), 3.68(\mathrm{~s}, 4 \mathrm{H}), 3.26(\mathrm{q}, J=8.8 \mathrm{~Hz}, 2 \mathrm{H}), 2.65(\mathrm{~d}, J=$ $5.6 \mathrm{~Hz}, 2 \mathrm{H}) .{ }^{13} \mathrm{C}-\mathrm{NMR}\left(\mathrm{CDCl}_{3}\right): \delta(\mathrm{ppm}) 195.92,176.77,175.24,159.32,148.90,147.76$, $143.07,136.90,136.51,130.96,130.25,129.88,129.38,127.62,127.08,121.77,117.69,105.43$ 36.70, 35.34, 28.47. HRMS expected for $\mathrm{C}_{32} \mathrm{H}_{22} \mathrm{~N}_{2} \mathrm{O}_{7} \mathrm{~S}_{2}: 611.0925$; found : 611.0927.

\section{6-Nitro-3-p-nitrophenylchromen-2-one (11)}

2-Hydroxy-5-nitrobenzaldehyde (1.00 g, $6.00 \mathrm{mmol})$ and p-nitrophenylacetic acid (1.08 g, 6.00 mmol) were placed in a dry $25-\mathrm{mL}$ round bottom flask. Acetic anhydride (11.2 $\mathrm{mL}, 120 \mathrm{mmol})$ was then added with stirring. To this suspension sodium hydride $(240 \mathrm{mg}, 6.00 \mathrm{mmol})$ was then 
added in small aliquots (60\% oil suspension) and the reaction mixture was stirred for $3 \mathrm{~h}$ at room temperature. The resulting beige powder was collected by filtration and washed with diethyl ether, then dried under vacuum to give compound $\mathbf{1 1}$ in $95 \%$ yield $(1.77 \mathrm{~g}, 5.67 \mathrm{mmol}) . \mathrm{mp}$ : $250{ }^{\circ} \mathrm{C}(\mathrm{dec}) . \quad$ FTIR $(\mathrm{KBr})\left(\mathrm{cm}^{-1}\right): 1620,1480,1750,1540,1350 .{ }^{1} \mathrm{H}-\mathrm{NMR}\left(\mathrm{DMSO}-\mathrm{d}_{6}\right): \delta$ (ppm) $8.80(\mathrm{~d}, J=2.6 \mathrm{~Hz}, 1 \mathrm{H}), 8.62(\mathrm{~s}, 1 \mathrm{H}), 8.47(\mathrm{dd}, J=9.1 \mathrm{~Hz}, J=2.7 \mathrm{~Hz}, 1 \mathrm{H}), 8.37(\mathrm{dd}, J=$ $8.9 \mathrm{~Hz}, J=2.0 \mathrm{~Hz}, 1 \mathrm{H}), 8.02(\mathrm{dd}, J=8.9 \mathrm{~Hz}, J=2.0 \mathrm{~Hz}, 2 \mathrm{H}), 7.71(\mathrm{~d}, J=9.1 \mathrm{~Hz}, 1 \mathrm{H}) .{ }^{13} \mathrm{C}-$ NMR $\left(\right.$ DMSO-d $\left._{6}\right): \delta(\mathrm{ppm}) 159.43,157.87,148.46,144.68,142.30,141.50,130.85,127.92$ 125.77, 124.51, 120.53, 118.64. HRMS : expected, 312.0382; found, 312.0391. Elemental analysis for $\mathrm{C}_{15} \mathrm{H}_{8} \mathrm{~N}_{2} \mathrm{O}_{6}$ : expected : C, 57.70; H, 2.58; N, 8.97; found : C, 57.37; H, 2.62; N, 8.78.

\section{6-Amino-3-p-aminophenylchromen-2-one (12)}

Following the same protocol used to obtain diamine 2, palladium on carbon (10\% water) $(0.322$ $\mathrm{g}, 1 / 10^{\text {th }}$ of the mass of $\left.\mathbf{1 1}\right)$ and a solution of sodium borohydride $(1.21 \mathrm{~g}, 32.9 \mathrm{mmol})$ in water $(22.6 \mathrm{~mL})$ were used to reduce a suspension of compound $\mathbf{1 1}(4.03 \mathrm{~g}, 12.9 \mathrm{mmol})$ in methanol (775 mL). In this way, compound $12(2.04 \mathrm{~g}, 8.1 \mathrm{mmol})$ was obtained as a yellow solid in $63 \%$ yield. FTIR $(\mathrm{KBr})\left(\mathrm{cm}^{-1}\right): 3180,3120,1700,1610,850 .{ }^{1} \mathrm{H}-\mathrm{NMR}\left(\right.$ DMSO-d $\left._{6}\right): \delta(\mathrm{ppm}) 7.84$ (s, 1H), $7.46(\mathrm{~d}, J=8.5 \mathrm{~Hz}, 2 \mathrm{H}), 7.08(\mathrm{~d}, J=8.6 \mathrm{~Hz}, 1 \mathrm{H}), 6.79(\mathrm{~d}, J=8.5 \mathrm{~Hz}, 1 \mathrm{H}), 6.77(\mathrm{~s}, 1 \mathrm{H})$,

$6.60(\mathrm{~d}, J=7.8 \mathrm{~Hz}, 2 \mathrm{H}), 5.32(\mathrm{ds}, 3 \mathrm{H}), 5.15(\mathrm{ds}, 3 \mathrm{H}) .{ }^{13} \mathrm{C}-\mathrm{NMR}\left(\right.$ DMSO-d $\left._{6}\right): \delta(\mathrm{ppm}) 163.34$, $150.20,146.43,145.43,137.99,130.38,127.88,123.18,11.33,118.85,116.94,114.39,111.04$. HRMS : expected , 252.0899; found, 252.0898. Elemental analysis for $\mathrm{C}_{15} \mathrm{H}_{12} \mathrm{~N}_{2} \mathrm{O}_{2}$ : expected : C, 71.42; H, 4.79; N, 11.10; found : C, 71.30; H, 4.58; N, 10.92 . 


\section{6-Maleimidyl-3-p-maleimidylphenylchromen-2-one (13)}

The protocol used to obtain compound 4 was followed, using diamine 12 (400 mg, $1.59 \mathrm{mmol}$ ) and maleic anhydride (777 $\mathrm{mg}, 4.77 \mathrm{mmol})$ in $15 \mathrm{~mL}$ chloroform and then $15 \mathrm{~mL}$ acetic anhydride $(0.16 \mathrm{~mol})$ and $130 \mathrm{mg}$ sodium acetate $(1.59 \mathrm{mmol})$. In this way, $\mathbf{1 3}$ was obtained as a beige solid and dried over vacuum to give 13 in $20 \%$ yield $(131 \mathrm{mg}, 0.32 \mathrm{mmol}) . \mathrm{mp}:>250{ }^{\circ} \mathrm{C}$ (dec). FTIR $(\mathrm{KBr})\left(\mathrm{cm}^{-1}\right): 2923,1721,1615,1520,1347,853 .{ }^{1} \mathrm{H}-\mathrm{NMR}\left(\mathrm{DMSO}_{\mathrm{d}}\right): \delta(\mathrm{ppm})$ $8.36(\mathrm{~s}, 1 \mathrm{H}), 7.87(\mathrm{dd}, J=8.9 \mathrm{~Hz}, J=2.0 \mathrm{~Hz}, 2 \mathrm{H}), 7.85(\mathrm{~d}, J=8.6 \mathrm{~Hz}, 1 \mathrm{H}), 7.60(\mathrm{dd}, J=8.6 \mathrm{~Hz}$, $J=2.6 \mathrm{~Hz}, 1 \mathrm{H}), 7.59(\mathrm{~d}, J=2.6 \mathrm{~Hz}, 1 \mathrm{H}), 7.45(\mathrm{dd}, J=8.9 \mathrm{~Hz}, J=2.0 \mathrm{~Hz}, 2 \mathrm{H}), 7.24(\mathrm{~d}, 4 \mathrm{H})$. ${ }^{13} \mathrm{C}-\mathrm{NMR}\left(\mathrm{DMSO}-\mathrm{d}_{6}\right): \delta(\mathrm{ppm}) 169.94,159.60,152.04,141.00,134.90,133.82,131.96,130.50$, 130.11, 129.25, 127.88, 126.95, 126.50, 119.77, 117.40, 104.30. HRMS : expected, 412.0695; found, 412.0695. Elemental analysis for $\mathrm{C}_{23} \mathrm{H}_{12} \mathrm{~N}_{2} \mathrm{O}_{6}$ : expected: $\mathrm{C}, 66.99 ; \mathrm{H}, 2.93 ; \mathrm{N}, 6.79$; found: $\mathrm{C}, 66.13 ; \mathrm{H}, 2.84 ; \mathrm{N}, 6.64$.

\section{6-(3'-Ethylthiosuccinimidyl)-3-p-(3'-ethylthiosuccinimidyl)phenylchromen-2-one (14)}

To a solution of compound $13(20 \mathrm{mg}, 0.049 \mathrm{mmol})$ in $2.5 \mathrm{~mL}$ of dry DMSO was added $110 \mu \mathrm{L}$ of ethanethiol $(0.147 \mathrm{mmol})$ and the solution was allowed to stir overnight at room temperature. A $100-\mathrm{mL}$ aliquot of water was then added and the solution was extracted 4 times with $20 \mathrm{~mL}$ dichloromethane. The organic layers were combined, dried over $\mathrm{MgSO}_{4}$ and removed under reduced pressure, apart from traces of residual DMSO, giving compound $\mathbf{1 4}$ in $89 \%$ yield (22 mg, $0.044 \mathrm{mmol})$. FTIR $(\mathrm{KBr})\left(\mathrm{cm}^{-1}\right): 2950,1713,1602,835,756 .{ }^{1} \mathrm{H}-\mathrm{NMR}\left(\mathrm{DMSO}-\mathrm{d}_{6}\right): \delta$ (ppm $8.39(\mathrm{~s}, 1 \mathrm{H}), 7.86(\mathrm{~d}, J=8.5 \mathrm{~Hz}, 2 \mathrm{H}), 7.74(\mathrm{~d}, J=2.5 \mathrm{~Hz}, 1 \mathrm{H}), 7.60(\mathrm{~d}, J=9.1 \mathrm{~Hz}, 1 \mathrm{H})$, $7.55(\mathrm{dd}, J=8.9 \mathrm{~Hz}, J=2.5 \mathrm{~Hz}, 1 \mathrm{H}), 7.38(\mathrm{~d}, J=8.6 \mathrm{~Hz}, 2 \mathrm{H}), 4.17(\mathrm{~m}, 2 \mathrm{H}), 3.42(\mathrm{dd}, J=8.2$ $\mathrm{Hz}, J=2.0 \mathrm{~Hz}, 1 \mathrm{H}), 3.38(\mathrm{dd}, J=8.2 \mathrm{~Hz}, J=2.0 \mathrm{~Hz}, 1 \mathrm{H}), 2.84(\mathrm{~m}, 4 \mathrm{H}), 2.76(\mathrm{~d}, J=5.6 \mathrm{~Hz}$, 
$1 \mathrm{H}), 2.71(\mathrm{~d}, J=5.5 \mathrm{~Hz}, 1 \mathrm{H}), 1.25(\mathrm{t}, J=7.3 \mathrm{~Hz}, 6 \mathrm{H}) .{ }^{13} \mathrm{C}-\mathrm{NMR}\left(\mathrm{DMSO}-\mathrm{d}_{6}\right): \delta(\mathrm{ppm})$ $176.96,175.33,160.45,153.44,141.38,135.52,133.56,131.38,130.26,129.42,127.89,127.79$, 120.76, 117.79, 37.26, 25.82, 20.49, 19.31, 15.31. HRMS expected for $\mathrm{C}_{27} \mathrm{H}_{24} \mathrm{O}_{6} \mathrm{~S}_{2}$ : 537.1168; found: 537.1164 .

\section{1,5-Dimaleimidylnaphthalene (15)}

The protocol used to obtain compound 4 was followed, using a suspension of $300 \mathrm{mg}$ of 1,5diaminonaphtalene $(1.90 \mathrm{mmol})$ in $20 \mathrm{~mL}$ of dry chloroform, $560 \mathrm{mg}(5.70 \mathrm{mmol})$ maleic anhydride, and then $15 \mathrm{~mL}$ acetic anhydride and $59 \mathrm{mg}(0.70 \mathrm{mmol})$ of sodium acetate. Compound 15 was thus obtained as a yellow solid in $71 \%$ yield $(428 \mathrm{mg}, 1.35 \mathrm{mmol}) . \mathrm{mp}>230$ ${ }^{\circ} \mathrm{C}(\mathrm{dec})$. FTIR $(\mathrm{KBr})\left(\mathrm{cm}^{-1}\right): 3070,1705 .{ }^{1} \mathrm{H}-\mathrm{NMR}\left(\right.$ DMSO-d $\left._{6}\right): \delta(\mathrm{ppm}) 7.87(\mathrm{~d}, J=7.80 \mathrm{~Hz}$, 1H), $7.70(\mathrm{t}, J=8.49 \mathrm{~Hz}, 1 \mathrm{H}), 7.62(\mathrm{~d}, J=7.38 \mathrm{~Hz}, 1 \mathrm{H}), 7.32(\mathrm{~s}, 4 \mathrm{H}) .{ }^{13} \mathrm{C}-\mathrm{NMR}\left(\mathrm{DMSO}-\mathrm{d}_{6}\right): \delta$ (ppm) 170.03, 134.61, 130.70, 128.24, 127.62, 126.31, 123.83. HRMS expected: 318.0641; found : 318.0641. Elemental analysis for $\mathrm{C}_{8} \mathrm{H}_{10} \mathrm{~N}_{2} \mathrm{O}_{4}$ expected: $\mathrm{C}, 67.93 ; \mathrm{H}, 3.17 ; \mathrm{N}, 8.80$; found: C, 67.05; H, 3.13; N, 8.62.

\section{1,5-Di(3'-ethylthiosuccinimidyl)naphthalene (16)}

The same protocol used to obtain $\mathbf{1 4}$ was followed, using $38 \mathrm{mg}$ of compound $\mathbf{1 5}(0.12 \mathrm{mmol})$ in $3 \mathrm{~mL}$ anhydrous DMSO (3 mL) with $27 \mu \mathrm{L}$ of ethanethiol $(0.36 \mathrm{mmol})$ to give compound $\mathbf{1 6}$ in $87 \%$ yield $(46 \mathrm{mg}, 0.10 \mathrm{mmol})$. FTIR $(\mathrm{KBr})\left(\mathrm{cm}^{-1}\right): 3000,1620,600 .{ }^{1} \mathrm{H}-\mathrm{NMR}\left(\mathrm{DMSO}-\mathrm{d}_{6}\right): \delta$ (ppm) $8.00(\mathrm{~d}, J=8.16 \mathrm{~Hz}, 1 \mathrm{H}), 7.78(\mathrm{t}, J=7.82 \mathrm{~Hz}, 1 \mathrm{H}), 7.75(\mathrm{~m}, 2 \mathrm{H}), 7.57(\mathrm{~d}, J=8.04 \mathrm{~Hz}$, $1 \mathrm{H}), 7.51(\mathrm{~d}, J=8.02 \mathrm{~Hz}, 2 \mathrm{H}), 4.42(\mathrm{~d}, J=5.20 \mathrm{~Hz}, 1 \mathrm{H}), 4.22(\mathrm{~d}, J=5.00 \mathrm{~Hz}, 1 \mathrm{H}), 3.64(\mathrm{~m}$, 1H), $3.42(\mathrm{~m}, 1 \mathrm{H}), 3.34(\mathrm{~m}, 6 \mathrm{H}), 1.89(\mathrm{t}, 6 \mathrm{H}) .{ }^{13} \mathrm{C}-\mathrm{NMR}\left(\mathrm{DMSO}_{\mathrm{d}}\right): \delta(\mathrm{ppm})$ 175.95, 174.40, 
$162.92,129.81,129.56,128.99,128.84,128.35,127.06,126.63,126.31,125.82,124.01,122.48$, 36.43, 35.91, 24.70, 24.40, 13.86. HRMS expected for $\mathrm{C}_{22} \mathrm{H}_{22} \mathrm{~N}_{2} \mathrm{O}_{4} \mathrm{~S}_{2}$ : 442.1021; found: 442.1023.

\section{3,6-Dinitro-1,8-naphthalic anhydride $(17)^{3}$}

Sulfuric acid $(40.0 \mathrm{~mL}, 0.75 \mathrm{~mol})$ was placed in a $100-\mathrm{mL}$ flask to which was added 1,8 naphtalic anhydride $(10.0 \mathrm{~g}, 50.5 \mathrm{mmol})$. The solution was cooled to $5{ }^{\circ} \mathrm{C}$ in an ice bath. Nitric acid $(10 \mathrm{~mL}, 0.22 \mathrm{~mol})$ was added drop by drop, taking care to not allow the temperature to exceed $20{ }^{\circ} \mathrm{C}$. The solution was then heated to $60{ }^{\circ} \mathrm{C}$ for $90 \mathrm{~min}$ and then cooled again to $4{ }^{\circ} \mathrm{C}$ for $24 \mathrm{~h}$ to induce precipitation. The resulting precipitate was removed by filtration and washed with cold glacial acetic acid. The solid was then washed with toluene $(3 \times 50 \mathrm{~mL})$ that was subsequently removed by rotary evaporation, repeating this process two more times to remove trace acetic acid, giving $\mathbf{1 7}$ as a beige powder (10.31g, $37.8 \mathrm{mmol})$ in $72 \%$ yield. mp 208-210 ${ }^{\circ} \mathrm{C}\left(\mathrm{lit}^{3} 208{ }^{\circ} \mathrm{C}\right.$. FTIR $(\mathrm{KBr})\left(\mathrm{cm}^{-1}\right): 3098,2897,1707,1617,1552,1336,1260 .{ }^{1} \mathrm{H}-\mathrm{NMR}$ (acetone-d $): \delta(\mathrm{ppm}) 9.80(\mathrm{~d}, J=2.23 \mathrm{~Hz}, 2 \mathrm{H}), 9.05(\mathrm{~d}, J=2.13 \mathrm{~Hz}, 2 \mathrm{H}) .{ }^{13} \mathrm{C}-\mathrm{NMR}$ (DMSO$\left.\mathrm{d}_{6}\right): \delta(\mathrm{ppm}) 159.75,148.14,134.62,133.28,131.62,128.06,123.07 . \quad$ HRMS expected : $288.0019 ;$ found : 288.0014 .

\section{$N$-(3,6-Dinitro-1,8-naphthalyl)-L-aspartic acid dimethyl ester (18)}

Compound 17 (300 mg, $1.06 \mathrm{mmol}$ ) and L-aspartic acid dimethyl ester hydrochloride (217 mg, $1.06 \mathrm{mmol}$ ) were added to a $25-\mathrm{mL}$ flask containing $10 \mathrm{~mL}$ of dry acetonitrile. Triethylamine $(180 \mu \mathrm{L}, 1.27 \mathrm{mmol})$ was added drop by drop to give a violet solution that was heated to $90{ }^{\circ} \mathrm{C}$ for $60 \mathrm{~h}$. After cooling the solution to room temperature, the solvent was removed through 
rotary evaporation and the solid obtained was purified by flash chromatography (70:30 hexane :EtOAc). The solvent was evaporated to give compound $\mathbf{1 8}$ as a beige powder ( $229 \mathrm{mg}$, $0.53 \mathrm{mmol})$ in $50 \%$ yield. $\mathrm{mp}: 128-129^{\circ} \mathrm{C}$. FTIR $(\mathrm{KBr})\left(\mathrm{cm}^{-1}\right): 3083,2954,1740,1678,1536$, 1319, 748. ${ }^{1} \mathrm{H}-\mathrm{NMR}\left(\mathrm{CDCl}_{3}\right): \delta(\mathrm{ppm}) 9.44(\mathrm{~d}, J=2.08 \mathrm{~Hz}, 2 \mathrm{H}), 9.39(\mathrm{~d}, J=2.07 \mathrm{~Hz}, 2 \mathrm{H})$, $6.17(\mathrm{dd}, J=7.88 \mathrm{~Hz}, J=6.29 \mathrm{~Hz}, 1 \mathrm{H}), 3.72(\mathrm{~s}, 3 \mathrm{H}), 3.63(\mathrm{~s}, 3 \mathrm{H}), 3.44(\mathrm{dd}, J=16.84 \mathrm{~Hz}, J=$ $6.26 \mathrm{~Hz}, 1 \mathrm{H}), 3.01(\mathrm{dd}, J=16.84, J=7.94 \mathrm{~Hz}, 1 \mathrm{H}) .{ }^{13} \mathrm{C}-\mathrm{NMR}\left(\mathrm{CDCb}_{3}\right): \delta(\mathrm{ppm}) 170.53$, $168.37,160.99,147.46,131.97,130.90,130.80,127.58,124.54,52.98,52.00,50.12,33.35$. HRMS expected for $\mathrm{C}_{18} \mathrm{H}_{14} \mathrm{~N}_{3} \mathrm{O}_{10}$ : 432.0679; found : 432.0688.. $\alpha_{\mathrm{D}}=-33^{\circ}\left(\mathrm{c}=1, \mathrm{CHCl}_{3}\right)$.

\section{$N$-(3,6-Diamino-1,8-naphthalyl)-L-aspartic acid dimethyl ester (19)}

Compound 18 (220 mg, $0.51 \mathrm{mmol}$ ) was placed in a 50-mL flask and $20 \mathrm{~mL}$ of THF and palladium on carbon (10\% wet) $\left(22 \mathrm{mg}, 1 / 10^{\text {th }}\right.$ the mass of 18$)$ were added. The reaction mixture was then placed in a hydrogenation reactor and left under $150 \mathrm{psi}$ pressure for $24 \mathrm{~h}$. The mixture was then filtered through Celite and the filtrate was evaporated under reduced pressure. The residue was purified by flash chromatography (70:30 EtOAc: $\left.\mathrm{CHCl}_{3}\right)$. The solvent was then removed by rotary evaporation to give compound $19(92.5 \mathrm{mg}, 0.25 \mathrm{mmol})$ as an orange powder in $49 \%$ yield. mp : $175-177{ }^{\circ} \mathrm{C}$. FTIR $(\mathrm{KBr})\left(\mathrm{cm}^{-1}\right): 3380,1748,1625,1316,797 .{ }^{1} \mathrm{H}-\mathrm{NMR}$ $\left(\right.$ DMSO-d $\left._{6}\right): \delta(\mathrm{ppm}) 7.59(\mathrm{~d}, J=1.90 \mathrm{~Hz}, 2 \mathrm{H}), 6.97(\mathrm{~d}, J=1.90 \mathrm{~Hz}, 2 \mathrm{H}), 6.02(\mathrm{dd}, J=8.00 \mathrm{~Hz}$, $J=5.43 \mathrm{~Hz}, 1 \mathrm{H}), 5.74(\mathrm{~s}, 4 \mathrm{H}), 3.61(\mathrm{~s}, 6 \mathrm{H}), 3.28(\mathrm{dd}, J=16.49 \mathrm{~Hz}, J=8.59 \mathrm{~Hz}, 1 \mathrm{H}), 2.85(\mathrm{dd}, J$ $=16.53 \mathrm{~Hz}, J=5.00 \mathrm{~Hz}, 1 \mathrm{H}) .{ }^{13} \mathrm{C}-\mathrm{NMR}\left(\mathrm{DMSO}_{6}\right): \delta(\mathrm{ppm}) 171.81,170.66,164.56,148.76$, 136.60, 122.74, 118.43, 115.51, 111.18, 111.11, 53.50, 50.73, 49.52, 34.76. HRMS expected for $\mathrm{C}_{18} \mathrm{H}_{17} \mathrm{~N}_{3} \mathrm{O}_{6}: 371.1117$; found : 371.1135. $\alpha_{\mathrm{D}}=-150^{\circ}(\mathrm{c}=0.1, \mathrm{DMSO})$. 


\section{$N$-(3,6-Dimaleimidyl-1,8-naphthalyl)-L-aspartic acid dimethyl ester (20)}

Compound 19 (200 mg, $0.54 \mathrm{mmol})$ and maleic anyhdride $(159 \mathrm{mg}, 1.62 \mathrm{mmol})$ were placed in a dry $50-\mathrm{mL}$ round-bottom flask. Chloroform $(15 \mathrm{~mL})$ was added and the solution was heated to reflux for $24 \mathrm{~h}$. The solution was then cooled to room temperature and filtered. The brown precipitate thus recovered was dried under reduced pressure. The solid was then placed in a 25$\mathrm{mL}$ round-bottom flask to which sodium acetate $(21 \mathrm{mg}, 0.37 \mathrm{eq})$ and acetic anhydride $(10 \mathrm{~mL})$ were added. The solution was heated to $100{ }^{\circ} \mathrm{C}$ for $90 \mathrm{~min}$. The solvent was then removed through evaporation and the resulting solid was dissolved in ethyl acetate. The solution was cooled to $4^{\circ} \mathrm{C}$ for $48 \mathrm{~h}$ and the recovered precipitate was washed with $25 \mathrm{~mL}$ of $80: 20$ waterDMSO and then $25 \mathrm{~mL}$ of water. The solid was then dissolved in a minimal volume of hot ethyl acetate and hexane was added to induce precipitation of the expected product (20) (53 mg, 0.10 mmol) as an orange powder in $19 \%$ yield. $\mathrm{mp}>230{ }^{\circ} \mathrm{C}(\mathrm{dec})$. FTIR $(\mathrm{KBr})\left(\mathrm{cm}^{-1}\right): 3102,1717$, 1669, 1413. ${ }^{1} \mathrm{H}-\mathrm{NMR}\left(\mathrm{DMSO}_{\mathrm{d}}\right): \delta(\mathrm{ppm}) 8.60(\mathrm{~d}, J=1.83 \mathrm{~Hz}, 2 \mathrm{H}), 8.58(\mathrm{~d}, J=1.86 \mathrm{~Hz}, 2 \mathrm{H})$, $7.32(\mathrm{~s}, 4 \mathrm{H}), 6.12(\mathrm{dd}, J=7.96 \mathrm{~Hz}, J=5.38 \mathrm{~Hz}, 1 \mathrm{H}), 3.64(\mathrm{~s}, 3 \mathrm{H}), 3.63(\mathrm{~s}, 3 \mathrm{H}), 3.32(\mathrm{dd}, J=$ $16.52 \mathrm{~Hz}, J=8.60 \mathrm{~Hz}, 1 \mathrm{H}), 2.97(\mathrm{dd}, J=16.53 \mathrm{~Hz}, J=5.03 \mathrm{~Hz}, 1 \mathrm{H}) .{ }^{13} \mathrm{C}-\mathrm{NMR}\left(\mathrm{DMSO}-\mathrm{d}_{6}\right): \delta$ (ppm) 171.77, 170.63, 170.33, 163.45, 136.08, 132.70, 132.35, 132.16, 130.80, 125.85, 123.47, 53.72, 52.83, 50.16, 34.46. HRMS expected: 532.0992; found: 532.0980. $\alpha_{\mathrm{D}}=-85^{\circ}(\mathrm{c}=0.1$, DMSO).

\section{$N$-(3,6-Di(3'-ethylthiosuccinimidyl)-1,8-naphthalyl)-L-aspartic acid dimethyl ester (21)}

The reaction of $26 \mathrm{mg}(0.49 \mathrm{mmol})$ of compound 20 in $2.5 \mathrm{~mL}$ dry DMSO with $110 \mu \mathrm{L}$ ethanethiol $(0.147 \mathrm{mmol})$ was carried out according to the same protocol used to obtain compound 7, giving compound 21 in $89 \%$ yield $(29 \mathrm{mg}, 0.044 \mathrm{mmol})$. FTIR $(\mathrm{KBr})\left(\mathrm{cm}^{-1}\right)$ : 
3104, 1715, 1671, 1528, 756. ${ }^{1} \mathrm{H}-\mathrm{NMR}\left(\mathrm{DMSO}_{\mathrm{d}}\right): \delta(\mathrm{ppm}) 8.59(\mathrm{~d}, J=1.82 \mathrm{~Hz}, 2 \mathrm{H}), 8.52$ $(\mathrm{d}, J=1.78 \mathrm{~Hz}, 2 \mathrm{H}), 6.08(\mathrm{dd}, J=7.86, J=5.29 \mathrm{~Hz}, 1 \mathrm{H}), 4.20(\mathrm{q}, J=8.66 \mathrm{~Hz}, 2 \mathrm{H}), 3.61(\mathrm{~m}, 10$ H), $3.41(\mathrm{dd}, J=16.46, J=8.56 \mathrm{~Hz}, 1 \mathrm{H}), 2.88(\mathrm{~m}, 5 \mathrm{H}), 1.25(\mathrm{t}, J=8.27 \mathrm{~Hz}, 6 \mathrm{H}) .{ }^{13} \mathrm{C}-\mathrm{NMR}$ $\left(\mathrm{DMSO}-\mathrm{d}_{6}\right): \delta$ (ppm) 175.86, 173.73, 170.26, 169.15, 168.80, 161.87, 134.58, 131.96, 131.20, $130.68,129.30,127.72,127.34,126.98,124.74,124.46,121.98,121.73,52.18,51.32,48.64$, 32.93, 24.44, 18.98, 17.78, 13.84. HRMS expected for $\mathrm{C}_{30} \mathrm{H}_{29} \mathrm{O}_{10} \mathrm{~S}_{2}$ : 655.1294; found: 655.1290. 


\section{Scheme 1}<smiles>CCCCNc1cc2cc(N)ccc2oc1=O</smiles><smiles>[R]C1=CC(=O)N(c2ccc3oc(=O)c(N4C(=O)C=C([R])C4=O)cc3c2)C1=O</smiles><smiles>Cc1cc2oc(=O)c(N)cc2cc1N</smiles>

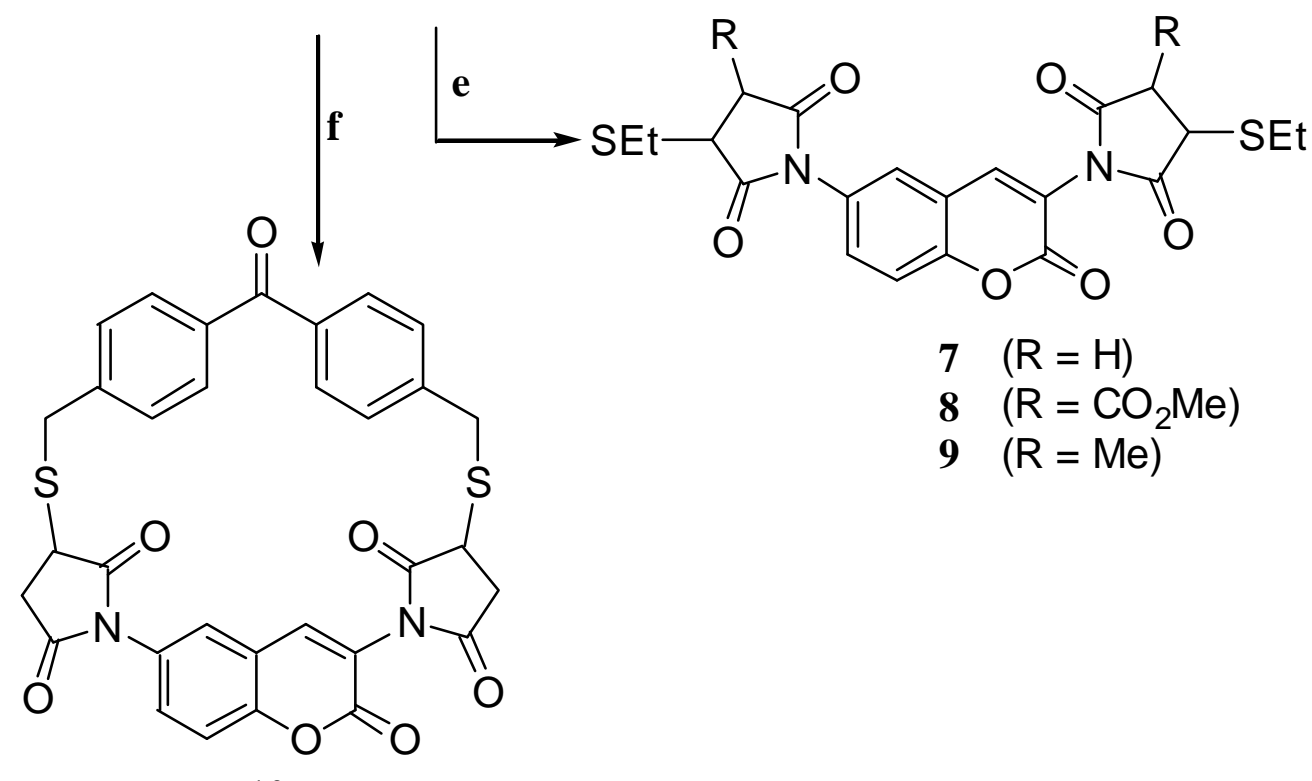

10

(a) $\mathrm{N}$-acetylglycine, $\mathrm{NaH}, \mathrm{Ac}_{2} \mathrm{O}, \mathrm{rt}, 61 \%$; (b) $\mathrm{Pd} / \mathrm{C}\left(10 \% \mathrm{H}_{2} \mathrm{O}\right), \mathrm{NaBH}_{4}, \mathrm{MeOH}, 57 \%$; (c) EtOH, $\mathrm{HCl}$, reflux, 65\%; (d) (i) maleic anhydride, $\mathrm{CHCl}_{3}$, reflux, (ii) $\mathrm{Ac}_{2} \mathrm{O}, \mathrm{AcONa}, 100{ }^{\circ} \mathrm{C}, 20 \%(2$ steps); (e) EtSH, $\mathrm{CHCl}_{3}$ or DMSO, rt, 83-85\%; (f) $\mathrm{BMMB}, \mathrm{NEt}_{3}, \mathrm{CHCl}_{3}$, reflux, $78 \%$. 


\section{Scheme 2}

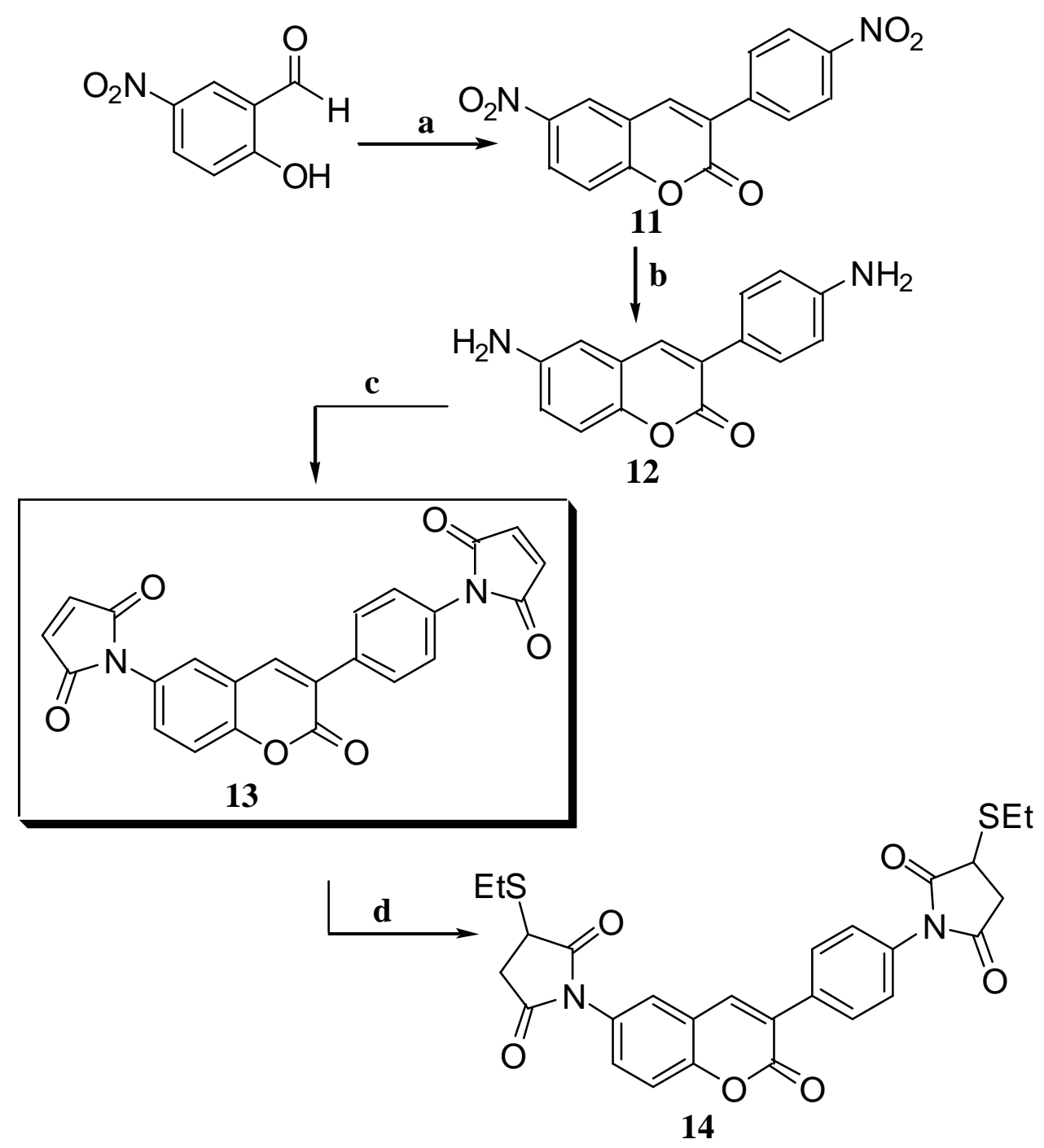

(a) 4-nitrophenylacetic acid, $\mathrm{Ac}_{2} \mathrm{O}, \mathrm{NaH}, \mathrm{rt}, 95 \%$; (b) $\mathrm{Pd} / \mathrm{C}\left(10 \% \mathrm{H}_{2} \mathrm{O}\right), \mathrm{NaBH}_{4}, \mathrm{MeOH}, 63 \%$; (c)

(i) maleic anhydride, $\mathrm{CHCl}_{3}$, reflux, (ii) $\mathrm{Ac}_{2} \mathrm{O}, \mathrm{AcONa}, 100^{\circ} \mathrm{C}, 20 \%$ (2 steps) (d) $\mathrm{EtSH}, \mathrm{DMSO}$, rt, $89 \%$. 
Scheme 3
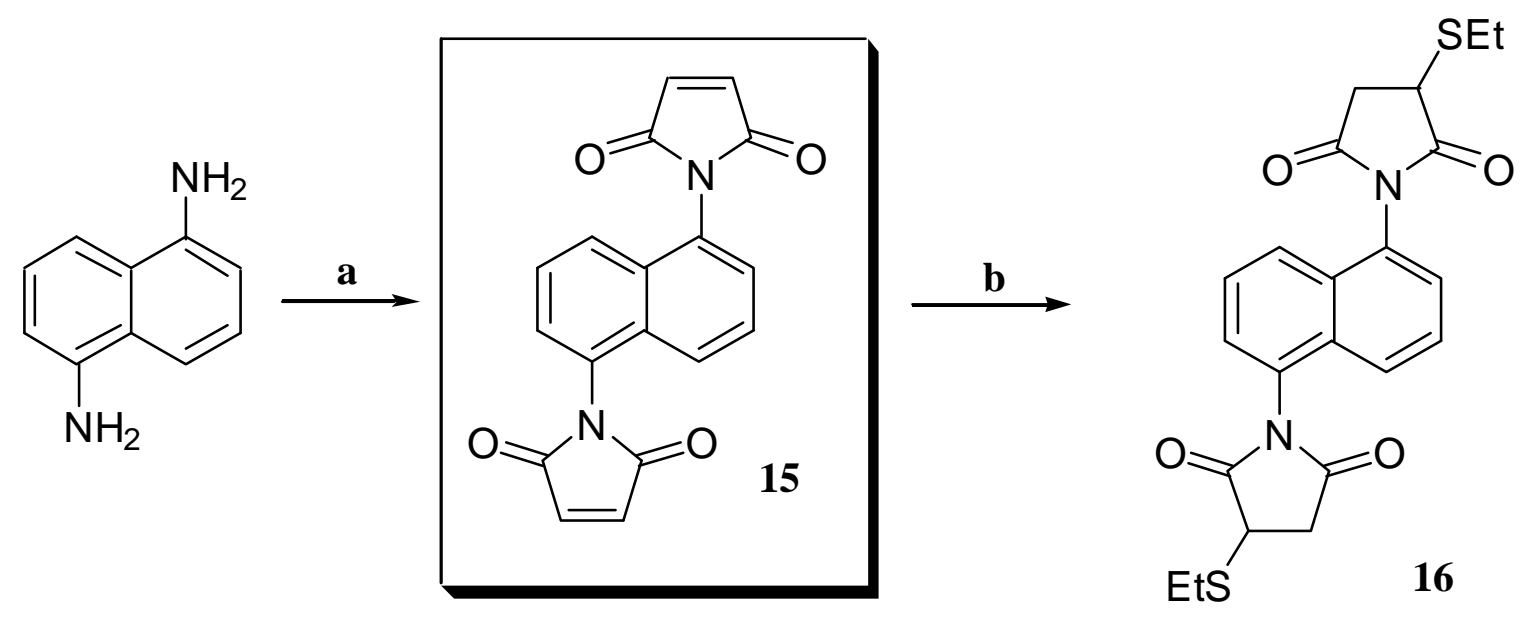

(a) (i) maleic anhydride, $\mathrm{CHCl}_{3}$, reflux, (ii) $\mathrm{Ac}_{2} \mathrm{O}, \mathrm{AcONa}, 100{ }^{\circ} \mathrm{C}, 75 \%$ (2 steps) (b) $\mathrm{EtSH}$, DMSO, rt, 87\%. 


\section{Scheme 4}<smiles>COC(=O)C[C@H](C(=O)OC)N1C(=O)c2cc([N+](=O)[O-])cc3cc([N+](=O)[O-])cc(c23)C1=O</smiles>

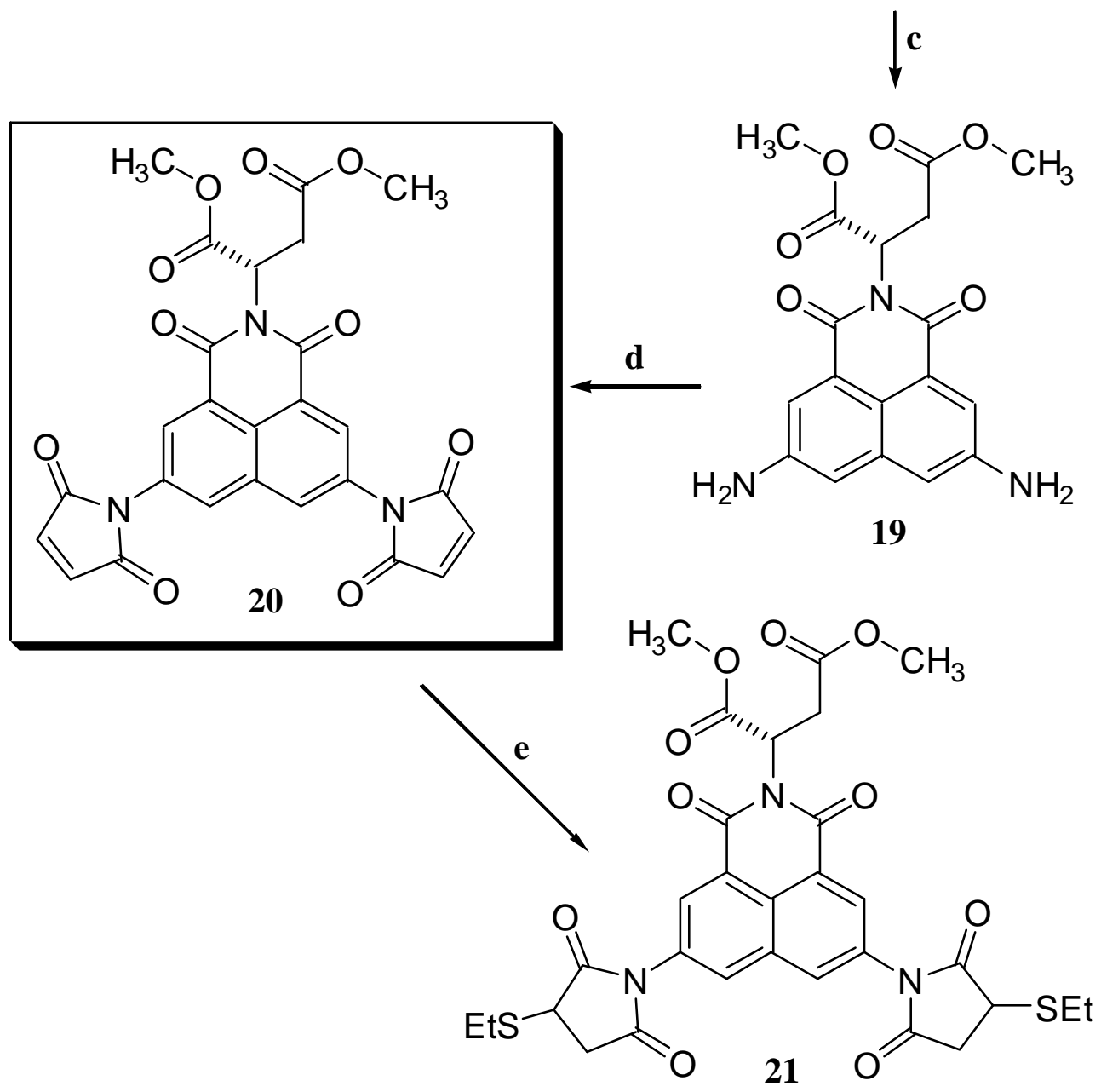

(a) $\mathrm{H}_{2} \mathrm{SO}_{4}, \mathrm{HNO}_{3}, 10-20{ }^{\circ} \mathrm{C}, 72 \%$ (b) $\mathrm{CH}_{3} \mathrm{CN}$, TEA, L-aspartic acid dimethyl ester $\bullet \mathrm{HCl}, 90{ }^{\circ} \mathrm{C}$, $45 \%$ (c) $\mathrm{H}_{2} / \mathrm{Pd} / \mathrm{C}, 150 \mathrm{psi}, \mathrm{THF}, 49 \%$ (d) (i) maleic anhydride, $\mathrm{CHCl}_{3}$, reflux, (ii) $\mathrm{Ac}_{2} \mathrm{O}, \mathrm{AcONa}$ $100{ }^{\circ} \mathrm{C}, 19 \%$ (2 steps). (e) EtSH, DMSO, rt, $89 \%$. 


\section{References}

1 Trivedi, K.; Sethna, S. J. Org. Chem. 1960, 25, 1817.

2 Kokotos, G.; Tzougrakic, C. J. Heterocyclic Chem. 1986, 23, 87.

3 Braña, M. F.; Castellano, J. M.; Morán, M.; Pérez de Vega, M. J.; Romerdahl, C. R.; Qian, X.-D.; Bousquet, P.; Emiling, F.; Schlick, E.; Keilhauer, G. Anti-cancer Drug Design 1993, 8, 257. 